INPLASY

PROTOCOL

To cite: Hulme et al. Deaf Sign

Language users and Audiology

Services: A scoping review on

cultural competence. Inplasy

protocol 202210133. doi:

10.37766/inplasy2022.1.0133

Received: 29 January 2022

Published: 29 January 2022

Corresponding author:

Celia Hulme

celia.hulme@postgrad.manchester.ac.uk

Author Affiliation:

University of Manchester

Support: NIHR MBRC Doctoral

Fellowship.

Review Stage at time of this submission: Preliminary searches.

Conflicts of interest:

None declared.

\section{Deaf Sign Language users and Audiology Services: A scoping review on cultural competence}

Hulme, C1; Young, A2; Rogers, K³; Munro, KJ4.

Review question / Objective: This study aims to identify culturally competent practice in audiology services from service provider and adult Deaf sign language users' perspectives. Therefore, the questions are as follows: (1) Are audiology services providing culturally competent practice to adult patients who are Deaf sign language users? (2) What are adult Deaf sign language users' experiences of audiology services from the perspective of cultural competence?

Information sources: The following databases will be used: PubMed, Embase, CINHAL, PsychlnFO, Web of Science SSCI and Project Muse. Grey literature (for example, guidelines, policies, and practice documents) will be searched. Also, key journals, reference lists and grey literature will be searched for additional references. There will be no publication date restriction to avoid excluding papers identified in non-indexed papers. The search date for each database and platform will be reported.

INPLASY registration number: This protocol was registered with the International Platform of Registered Systematic Review and Meta-Analysis Protocols (INPLASY) on 29 January 2022 and was last updated on 29 January 2022 (registration number INPLASY202210133).

\section{INTRODUCTION}

Review question / Objective: This study aims to identify culturally competent practice in audiology services from service provider and adult Deaf sign language users' perspectives. Therefore, the questions are as follows: (1) Are audiology services providing culturally competent practice to adult patients who are Deaf sign language users? (2) What are adult Deaf sign language users' experiences of audiology services from the perspective of cultural competence?

Rationale: To date, knowledge about culturally competent audiology services and Deaf sign language users is lacking. There is a paucity of evidence internationally about Deaf sign language 
users who access hearing aid services and of adult hearing aid services responses to addressing effective engagement with Deaf sign language users from a cultural and linguistic perspective rather than from a medical and disability perspective of patient care. Additionally, no scoping review has been carried out relating to this topic. Therefore, the rationale for this scoping review is to identify and better understand current provisions for Deaf sign language users who access audiology services. The findings from this review would inform audiology services which could, in turn, improve their cultural competence practices with Deaf sign language users. The method for scoping review will follow Peters et al (2020) ninestep methodology framework for scoping reviews. This framework also dovetails with the PRISMA Scoping Review reporting checklist (Tricco et al., 2018).

Condition being studied: This scoping study concerns Deaf people who are sign language users in whatever country (e.g., users of BSL, British Sign Language in the UK; users of ASL, American Sign Language in the US) and who access audiology services (whether state or private providers). Sign language users who are Deaf are a cultural-linguistic minority distinct from those who lose their hearing later in life or are predominantly spokenlanguage users. They are perceived to be atypical users of audiology services. Also, guidance on cultural competence to audiology services tends also to overlook them focussing instead on patients who are users of spoken languages from minority cultural backgrounds. A scoping review with a specific focus on Deaf sign language users concerning service provision and their experiences of accessing and regularly using audiology services is needed to identify current best practices in cultural competence or where cultural competency practice is lacking and its likely consequences.

\section{METHODS}

Search strategy: PubMed, Embase, CINHAL, PsychINFO, Web of Science SSCI and Project Muse are the primary databases that will be searched. Additionally, grey literature (for example, guidelines, policies, and practice documents) will be searched. Finally, key journals, reference lists and grey literature will be searched for additional references. The search strategy will include the following headings or keywords: 1) descriptors of audiology services; 2) descriptors of deaf terms; 3) descriptors for cultural competence and 4) indicators. Boolean operators AND, NOT, OR will be applied and free text terms outlined in each heading. Truncation will be applied wherever possible (Deaf*, hard of hear*, hearing impair*, sign*, cultural comp*, program*, cultural*).

Participant or population: All literature that involves sign language, Deaf signing individuals or the Deaf community/ populations will be included in the review. Signed languages are used worldwide and each country has its own distinct sign language; therefore, relevant literature from other countries will be included in the review if written in English.

Intervention: Any study or reports of sign language, audiology services and cultural competence pertaining to Deaf people will be included in the review.

Comparator: Although not essential for this review, any studies that include comparators will be included.

Study designs to be included: There will be no restrictions on the types of study design eligible for inclusion in this review. Any publications that are not in written English will be excluded from the review.

Eligibility criteria: To be included in the review, studies/research have to include text relating to cultural competence and Deaf adults who are sign language users within an audiology service setting.

Information sources: The following databases will be used: PubMed, Embase, CINHAL, PsychInF0, Web of Science SSCI and Project Muse. Grey literature (for 
example, guidelines, policies, and practice documents) will be searched. Also, key journals, reference lists and grey literature will be searched for additional references. There will be no publication date restriction to avoid excluding papers identified in nonindexed papers. The search date for each database and platform will be reported.

Main outcome(s): The primary outcome of interest will be any reports of Deaf sign language users accessing audiology services and patient experience of using such services with an emphasis on cultural competence.

Data management: The management of the data will be done in two phases. Phase One is the screening of the title and abstract. Retrieved records from all databases will be exported to Endnote and the software will be used to remove duplicates automatically. Manual duplication will also be performed to ensure that all records are duplicate free. These records will then be exported to Rayyan for eligibility screening that all reviewers can access. The first reviewer $(\mathrm{CH})$ will initially select inclusion for the title and abstract, and the second reviewer (KR) will screen the identified records to crosscheck the accuracy. Any discrepancies in the data extraction between the two reviewers will be discussed. The third reviewer (AY) will only step in if no agreement is reached between the first two reviewers. Any discussions and extractions will be recorded.

Phase Two of the screening process is reviewing the full text of the eligible papers. The full-text screening will be carried out by two reviewers ( $\mathrm{CH}$ and $\mathrm{KR}$ ) and the third reviewer (AY) will step in if there are any disagreements. The Preferred Reporting Items for Systematic Review and MetaAnalyses Extension for Scoping Reviews PRISMA-ScR (Tricco et al., 2018) flow chart will summarise the selection process ensure transparency.

All identified data extracted from full-text articles will be recorded in a Microsoft Excel document. The characteristics of the identified articles will be categorised as follows: (1) authors, (2), year of publication, (3) publication type, (4) country, (5) participant demographics, (6) study design, (7) number of participants (if applicable), (8) inclusion/exclusion criteria, (9) intervention and (10) outcomes/results.

Quality assessment / Risk of bias analysis: Scoping reviews do not typically include quality assessments; however, the Crowe Critical Appraisal Tool [CCAT] (Crowe and Sheppard, 2011) tool will be applied as a light touch. It is based on suitability to appraise multiple research designs critically. The latest version [Version 1.4] (Crowe, 2013) contains eight categories with 98 descriptors which include scores. The CCAT assessment allows a person to make notes in each category before scoring. Scoring will not be used for this review as it is not required for a scoping review.

Strategy of data synthesis: The data will be narratively synthesised as it is anticipated that there will be few studies involving cultural competence in audiology services relating to sign language users.

Subgroup analysis: As this is a scoping review, there is no plan for subgroup analysis.

Sensitivity analysis: As this is a scoping review, there is no plan for sensitivity analysis.

Language: Only publications that are written in English will be included in the scoping review.

Country(ies) involved: United Kingdom.

Keywords: Deaf, Sign language, Audiology, Cultural competence.

Dissemination plans: The scoping review findings will be published in peer-reviewed journals and presented at conferences.

Contributions of each author:

Author 1 - Celia Hulme - CH developed and prepared the review protocol and will lead the selection and data extraction processes and the risk of bias assessment. 
$\mathrm{CH}$ will also prepare the manuscript of this review.

Email: celia.hulme@postgrad.manchester.ac.uk Author 2 - Alys Young - AY critically reviewed this protocol and is the third reviewer in case of any disagreements. AY will also critically review the manuscript of this review.

Email: alys.young@manchester.ac.uk

Author 3 - Katherine Rogers - KR critically reviewed this protocol and will also contribute to the selection and data extraction processes. KR will also critically review the manuscript of this review.

Email: katherine.rogers@manchester.ac.uk Author 4 - Kevin Munro - KM critically reviewed this protocol and will also critically review the manuscript of this review.

Email: kevin.j.munro@manchester.ac.uk 